
\title{
28 Research Soure \\ The gut microbiome and its potential role in paradoxical anaerobism in pupfishes of the Mojave Desert
}

\section{Shrikant S Bhute}

University of Nevada, Las Vegas

\section{Brisa Escobedo}

University of Nevada, Las Vegas

\section{Mina Haider}

"University of Phoenix"

\section{Yididya Mekonen}

University of Nevada, Las Vegas

\section{Dafhney Ferrer}

University of Nevada, Las Vegas

\section{Stanley D. Hillyard}

University of Nevada, Las Vegas

\section{Ariel D Friel}

University of Nevada, Las Vegas

\section{Frank van Breukelen}

University of Nevada, Las Vegas

Brian P. Hedlund ( $\nabla$ brian.hedlund@unlv.edu )

University of Nevada, Las Vegas https://orcid.org/0000-0001-8530-0448

\section{Research Article}

Keywords: Pupfish, ethanol metabolism, paradoxical anaerobism, gut microbiome, Cetobacterium

Posted Date: April 28th, 2020

DOI: https://doi.org/10.21203/rs.2.19358/v2

License: (c) (1) This work is licensed under a Creative Commons Attribution 4.0 International License. Read Full License

Version of Record: A version of this preprint was published at Animal Microbiome on May 19th, 2020. See the published version at https://doi.org/10.1186/s42523-020-00037-5. 


\section{Abstract}

Background Pupfish frequently enter paradoxical anaerobism in response to endogenously produced or exogenously supplied ethanol in a dose-dependent manner. To decipher the role of the gut microbiota in ethanol-associated paradoxical anaerobism, gut microbial communities were depleted using a cocktail of antibiotics and profiled using 16S rRNA gene sequencing.

Results Compared to the control group ( $n=12)$, microbiota-depleted fish $(n=12)$ spent more time in paradoxical anaerobism. Our analysis indicated that the bacterial phyla Proteobacteria, Fusobacteria, Bacteroidetes, Firmicutes, Actinobacteria, Patescibacteria, and Dependentiae dominated the pupfish gut, which is consistent with other fish gut microbiota. Although the gut microbial communities with and without antibiotic treatment were similarly diverse, they were distinct and the greatest contribution to the dissimilarity $(27.38 \%)$ was the common fish commensal Cetobacterium.

Conclusions This study reports the first characterization of gut microbial communities of pupfish and suggests the microbiome may play a critical role in regulating metabolic strategies that are critical for survival in extremes of temperature and oxygen concentration. We speculate that Cetobacterium, a primary fermenter, also consumes ethanol through secondary fermentation via an alcohol dehydrogenase and therefore regulates the transition from paradoxical anaerobism to aerobic respiration in fish. Given the wide distribution and abundance of Cetobacterium in warm-water fishes, this process may be of broad importance, and suggests that the microbiome be carefully considered for both conservation and aquaculture.

\section{Background}

The Mojave Desert in the Southwestern United States is a home to isolated systems of springs that are geothermally or ambiently heated to 28 to $33^{\circ} \mathrm{C}$ and are inhabited by pupfish in the genus Cyprinodon [1]. Previously, we demonstrated that two lineages of pupfish reared at $33^{\circ} \mathrm{C}$ frequently entered a state of paradoxical anaerobism [2,3]. For periods of as much as 149 minutes, these pupfish consumed negligible oxygen despite its availability. Instead, pupfish reared or acclimated to $33^{\circ} \mathrm{C}$ produced $\sim 7.3 \mathrm{X}$ more ethanol than their counterparts maintained at $28^{\circ} \mathrm{C}$. Pupfish spontaneously exited paradoxical anaerobism, indicating there are no lasting deleterious effects on mitochondrial function. Both 28- and 33 ${ }^{\circ} \mathrm{C}$-acclimated pupfish entered paradoxical anaerobism when exposed to exogenous ethanol in a dosedependent manner [2]. Although $28^{\circ} \mathrm{C}$-acclimated pupfish rarely spontaneously used paradoxical anaerobism (1/262 assayed fish measured for a $2 \mathrm{~h}$ period), exposure to $1 \%$ ethanol (vol/vol) resulted in seven out of 12 of the fish immediately entering paradoxical anaerobism. We proposed that ethanol metabolism and the resulting accumulation of acetaldehyde results in closure of voltage-dependent anion channels (VDACs) in living pupfish and ultimately, the phenotype of paradoxical anaerobism [2] (see discussion for details). If our model is correct, changes in ethanol metabolism should affect use of paradoxical anaerobism. Our earlier work measuring ethanol accumulation by $28{ }^{\circ} \mathrm{C}$ - and $33^{\circ} \mathrm{C}$ acclimated fish suggested a microbial interaction. Measuring ethanol accumulation in a sealed container 
with a fish required us to pre-boil aquarium water, rinse the fish, and ensure the fish did not defecate or swim vigorously during the assay; rapid reduction of exogenous ethanol in the water otherwise occurred [2].

In the present study, we explore the possibility that the pupfish gut microbiome plays a role in the regulation of paradoxical anaerobism. Specifically, if the microbiome served as a sink for endogenously produced ethanol, then reducing the autochthonous gut microbiome with antibiotic treatment would be predicted to enhance the expression of paradoxical anaerobism produced by exogenous exposure to ethanol. In this study, gut contents of fish treated with antibiotics and untreated controls were collected for analysis of microbial community diversity and composition, which were then related to the expression of paradoxical anaerobism.

\section{Results}

\section{Exposure to antibiotics led to increased time in paradoxical anaerobism}

Exposure to a cocktail of antibiotics (kanamycin, gentamicin, colistin, metronidazole, and vancomycin made fish more prone to entering paradoxical anaerobism (Fig.1). In Fig.1A, an example of paradoxical anaerobism use is provided. Immediately after introduction of $\sim 1 \%$ ethanol (vol/vol) to the aquarium water, the fish entered a prolonged period of negligible oxygen use. Pupfish exposed to antibiotics and ethanol spent $40.5 \pm 11.3 \%$ of the post-ethanol time in paradoxical anaerobism - approximately $3.6 \mathrm{X}$ longer than did control fish (Fig.1B). This difference was statistically significant (Welch's t-test, $\mathrm{p}$-value $=$ 0.037). All 12 antibiotic-treated, $28^{\circ} \mathrm{C}$-acclimated pupfish used some paradoxical anaerobism when exposed to $1 \%$ ethanol ( $\mathrm{vol} / \mathrm{vol})$, whereas 8 of the 12-control pupfish used paradoxical anaerobism, which is consistent with our previous results [2].

\section{Antibiotic treatment was associated with the changes in community composition}

Illumina sequencing yielded an average of 18,688 (range: 3,713 - 82,969) 16S rRNA gene sequences per fish gut sample (Supplementary Table 1). One of the samples, PUP9, from the antibiotic-treated group, was removed from further analysis due to low sequence count. The microbiomes were moderately diverse, with amplicon sequence variant (ASV) richness ranging from 11- 154 and Shannon diversity values ranging from $0.67-6.23$, and there was no significant difference in richness or diversity (Observed ASVs and Shannon index, Fig.2A; Table S1) between control and antibiotic-treated microbiomes (Welch's t-test, observed ASVs: $p=0.450$ and Shannon index: $p=0.897$ ). However, beta diversity analysis based on non-metric multidimensional scaling (NMDS) using Bray-Curtis dissimilarity indicated that antibiotic treatment changed the composition of the microbiome (Fig.2B). This was further demonstrated by ANOSIM (Analysis of Similarity) analysis $(R=0.469, p=0.001)$. Overall, the dominant phyla were Proteobacteria (29.9\% and 46.9\%), Fusobacteria (47.3\% and $2.0 \%)$, Bacteroidetes $(11.7 \%$ and $18.5 \%)$, Firmicutes (15\% and $13.8 \%$ ), Actinobacteria (4.8\% and 5.3\%), Patescibacteria (2.5\% and $4.1 \%)$, and Dependentiae (0.0\% and 2.3\%); numbers in parentheses indicate mean relative abundance of each phylum in control and antibiotic-treated groups, respectively. Out of the 23 phyla detected in all samples 
(Fig.2C), Proteobacteria, Bacteroidetes, Actinobacteria, Patescibacteria, and Dependentiae had higher relative abundance in the antibiotic-treated group (Welch's t-test, $\mathrm{p}<0.05$ ), whereas only Fusobacteria was significantly reduced due to antibiotic treatment (Welch's t-test, $p<0.05$ ).

SIMPER (SIMilarity of PERcentages) analysis was performed to determine the contribution made by specific ASVs to the observed dissimilarity between control and antibiotic-treated fish. SIMPER identified nine ASVs contributing to $50 \%$ of observed differences, of which Cetobacterium (Fusobacteria), Brevinema (Spirochaetes), Pseudomonas pseudomonaspeli (Proteobacteria), Ileibacterium (Firmicutes), Aeromonas (Proteobacteria), and Verrucomicrobiaceae (Verrucomicrobia) were enriched in the control group, while Escherichia-Shigella (Proteobacteria) and Flavobacterium (Bacteroidetes) ASVs were enriched in the antibiotic-treated group (Fig.3; Supplementary Table 2). ANCOM (Analysis of Composition of Microbiomes) ( $\mathrm{W}=719)$ suggested that the loss of Cetobacterium in antibiotic-treated fish as a strong indicator of community differences. Cetobacterium was represented by two different ASVs, designated Cetobacterium and Cetobacterium 1 (Fig.3), which represent either separate species or separate populations of a single species. However, the genetic distance between these ASVs cannot be accurately assessed by small $16 \mathrm{~S}$ rRNA gene fragments, alone. The Cetobacterium 1 ASV comprised $33 \%$ of the healthy pupfish gut microbiome, whereas the Cetobacterium ASV was present at low abundance; neither were detected in antibiotic-treated fish (Fig.3).

\section{Cetobacterium may be involved in ethanol metabolism}

To probe the possible mechanism of microbial mediation of paradoxical anaerobism, we identified microbial taxa contributing to ethanol metabolism by predicting the presence of alcohol dehydrogenase in the simulated metagenome using PICRUSt (Phylogenetic Investigation of Communities by Reconstruction of Unobserved States). We identified 129 ASVs that likely have an alcohol dehydrogenase gene (adh; KO: K00001). Notably, ASVs assigned to Cetobacterium were the dominant bacteria predicted to encode Adh in control fish, indicating the potential role of Cetobacterium in clearing ethanol reaching the gut environment in these fish (Fig.4). In contrast, Flavobacterium ASVs were the dominant bacteria to predicted to encode Adh in antibiotic-treated fish. Other taxa that contributed to the adh gene pool include members of the Actinobacteria (Bifidobacterium and Mycobacterium); Proteobacteria (Acinetobacter, Delftia, Polynucleobacter, and Pseudomonas); and Firmicutes.

\section{Discussion}

Voltage-dependent anion channels (VDACs) allow substrates to enter the outer mitochondrial membrane and are assumed to be open during normal metabolism [4-6]. This assumption has led many to believe that low molecular weight molecules pass freely through the outer mitochondrial membrane. The continuation of glycolysis is dependent on the availability of $\mathrm{NAD}^{+}$[7]. In mammals, the production of lactate allows for $\mathrm{NAD}^{+}$regeneration during anaerobic metabolism. Under hypoxia, goldfish, and other warm-acclimated fish, may also regenerate $\mathrm{NAD}^{+}$through production of ethanol $[2,8]$. For instance, anoxic exposure of goldfish to $12 \mathrm{~h}$ at $4^{\circ} \mathrm{C}$ results in excretion of $6.63 \mu \mathrm{mol}$ ethanol $\cdot \mathrm{g}^{-1}$ fish into the 
water and tissue accumulation of $4.58 \mu \mathrm{mol}$ ethanol $\cdot \mathrm{g}^{-1}$ fish. However, ethanol metabolism, resulting in the production of acetaldehyde, is also able to suppress oxygen consumption through closure of the VDACs [9]. A closed VDAC would limit substrate entry into the mitochondrion and thus, oxygen consumption. We believe that paradoxical anaerobism is the result of pupfish lapsing into this phenotype e.g., relatively placid pupfish produce some ethanol as part of their normal metabolism. The associated acetaldehyde closes some VDACs. While this phenotype is frequently manifested by oxygen consumption that is more variable and lower than what may be otherwise expected, we believe a positive feedback mechanism resulting in more and more closure of the VDACs results in the paradoxical anaerobism characterized by negligible oxygen consumption. Consistent with this model are data from $33^{\circ} \mathrm{C}$ acclimated pupfish demonstrating a much higher frequency of a state of variable oxygen consumption ( $20 \%$ of $33^{\circ} \mathrm{C}$-acclimated pupfish assayed for $2 \mathrm{~h}$ as compared to $<4 \%$ of $28{ }^{\circ} \mathrm{C}$-acclimated pupfish), lower than expected oxygen consumption, and lower aerobic scope (maximum oxygen consumption • $\left.\mathrm{rVO}_{2}^{-1}\right)[2,3]$. In other words, both 28 - and $33^{\circ} \mathrm{C}$-acclimated pupfish are likely using a mosaic of both paradoxical anaerobism and oxygen consumption. However, the use of paradoxical anaerobism is more extensive in the $33^{\circ} \mathrm{C}$-acclimated pupfish than their $28^{\circ} \mathrm{C}$-acclimated counterparts. As a result, these pupfish are more likely to lapse into extensive paradoxical anaerobism use characterized by negligible oxygen consumption.

We hypothesized that the gut microbiome would serve as a sink for ethanol since ethanol readily crosses membranes. Consistent with our model is the finding here that pupfish exposed to antibiotics and ethanol experienced 3.6X more paradoxical anaerobism than did the control fish (Fig.1B). We contend that depletion of the microbiome results in more circulating ethanol in the pupfish and therefore longer bouts of paradoxical anaerobism. To gain insight into microorganisms that might be responsible for ethanol consumption, we characterized the gut microbial communities of pupfish treated with the antibiotic cocktail and compared them to control fish. Consistent with other fish gut microbiomes, the pupfish microbiome was dominated by members of the Proteobacteria, Fusobacteria, Bacteroidetes, Firmicutes, and Actinobacteria [10]. Our results indicate significant differences in microbial communities between the control and antibiotic-treated pupfish populations, which correlated with the increase in paradoxical anaerobism in the antibiotic-treated fish.

Prophylactic use of antibiotics during fish farming has been linked with increased abundance of antibiotic-resistant members of Bacteroidetes and Proteobacteria [11, 12], which is consistent with our observation of increased abundance of Bacteroidetes (Flavobacterium) and members of Proteobacteria, specifically Enterobacteriaceae, in antibiotic-treated pupfish. Species of the genus Flavobacterium ( $F$. psychrophilum, F. columnare, and F. branchiophilum) have been described in localized and systemic infections and implicated in mortality of both wild and aquaculture fish [13]. Antibiotic-resistant Flavobacterium isolates have also been obtained from fish farms [14], broadly consistent with their increased abundance in this study.

Elimination of Fusobacteria, more specifically Cetobacterium, from the antibiotic-treated fish indicates general sensitivity of this microorganism to the antibiotic cocktail used in this study. Cetobacterium ceti 
and other species of the phylum Fusobacteria are broadly sensitive to antibiotics, with the notable exception of vancomycin [15]. Cetobacterium is a microaerotolerant primary fermenter of carbohydrates and peptides, producing acetate as the major fermentation product, and is particularly abundant in warmwater fishes such as tilapia [16], carp [17], arapaima [18], catfish, and bass [19], comprising $>70 \%$ of the gut microbial community in many individuals. Isolates of Cetobacterium produce large quantities of vitamin B12 and is speculated to therefore promote fish health [20]. However, we suggest an additional hypothesis that Cetobacterium may play a role in regulating ethanol toxicity and the balance between paradoxical anaerobism and aerobic respiration in fish that are often limited for dissolved oxygen (Fig.5).

Several pathways for microbial ethanol consumption are known [21, 22], but all likely involve use of some form of Adh. Our finding that Cetobacterium is the dominant organism predicted to encode Adh in healthy pupfish and its absence in antibiotic-treated fish with prolonged bouts of paradoxical anaerobism is consistent with this alternative hypothesis; however, a rigorous test of this would require a direct test, for example by applying the principles of Koch's postulates.

Of direct relevance to active conservation efforts with pupfish, our surveys of planktonic cells in springs across Ash Meadows National Wildlife Refuge show that low-abundance populations of Cetobacterium are ubiquitous, which is consistent with a separate report of low-abundance planktonic Cetobacterium in Devils Hole [23]. In contrast, Cetobacterium is absent from nearby springs without fish populations. These planktonic bacteria are likely indicators that Cetobacterium is present in the guts of inhabitant pupfish and serve as inoculum for developing fish. Other work has suggested that Cetobacterium may be a target for probiotic development for fish well-being $[19,24]$. Our work suggests that Cetobacterium might be important for the development and health of pupfish and should be considered within the context of the current practice of antibiotic treatment of pupfish eggs at the AMFCF [34].

\section{Conclusion}

Our work catalogues the gut microbial communities of pupfish of the Mojave Desert and proposes its involvement in the paradoxical anaerobism observed in these fish. We show that removal of dominant gut flora, including members of genus Cetobacterium using antibiotics leads to increased duration of paradoxical anaerobism which could be linked with the ethanol utilizing abilities of Cetobacterium through the action of alcohol dehydrogenase (Fig.4). We further propose that regular microbiome monitoring of Ash Meadow Fish Conservation Facility (AMFCF) to detect the presence of Cetobacterium in the refuge tank, and the fish living in it, may help the active conservation of pupfish.

\section{Methods}

\section{Fish husbandry and antibiotic treatment}

Refuge pupfish that have been described as Cyprinado diabolis and Cyprinadon nevadensis mionectes hybrids [35] were maintained at $28^{\circ} \mathrm{C}$ as described previously [25]. Pupfish were either left untreated $(\mathrm{n}=$ $12)$ or exposed $(n=12)$ to a cocktail of kanamycin $\left(400 \mu \mathrm{g} \cdot \mathrm{ml}^{-1}\right)$, gentamicin $\left(35 \mu \mathrm{g} \cdot \mathrm{ml}^{-1}\right)$, colistin (850 
$\left.\mathrm{IU} \cdot \mathrm{ml}^{-1}\right)$, metronidazole $\left(215 \mu \mathrm{g} \cdot \mathrm{ml}^{-1}\right)$, and vancomycin $\left(45 \mu \mathrm{g} \cdot \mathrm{ml}^{-1}\right)$ for $48 \mathrm{~h}$ prior to oxygen consumption measurement. This combination of broad-spectrum antibiotics acting on protein synthesis (kanamycin and gentamicin), cell membrane integrity (colistin), DNA synthesis (metronidazole) and peptidoglycan synthesis (vancomycin) is often used to deplete the mouse gut microbial community to study Clostridioides difficile infections and is highly effective [26].

\section{Measurement of oxygen consumption after ethanol treatment}

Routine rates of oxygen consumption $\left(\mathrm{rVO}_{2}\right)$ were measured in fish showing minimal activity using flowthrough respirometry (Fig.S1)[2,3]. Briefly, four fish at a time were placed individually in glass metabolic chambers ( $60 \mathrm{~mL}$ volume) that were connected to flow cells with Clark-type oxygen electrodes (Strathkelvin, Scotland). The apparatus was placed in a $\sim 40 \mathrm{~L}$ aerated and heated $\left(28^{\circ} \mathrm{C}\right)$ aquarium. Water was drawn into the metabolic chambers from the surrounding aquarium and through the flow cells using a peristaltic pump. Oxygen consumption was calculated with Strathkelvin software for flowthrough respirometry using the appropriate temperature and pressure corrections. Flow rates were adjusted, according to the size of the fish, to maintain the excurrent $\mathrm{PO}_{2}$ above $100 \mathrm{~mm} \mathrm{Hg}(13.32 \mathrm{kPa}$; and well above the critical $\mathrm{PO}_{2}$ value of $47 \mathrm{~mm} \mathrm{Hg}$ that would otherwise limit oxygen consumption) [2]. These flow rates were $\sim 6$ to $8.4 \mathrm{~mL} \cdot \mathrm{min}^{-1}$.

Each trial began with a period of $\sim 60$ min after fish were placed in the chambers to allow $\mathrm{rVO}_{2}$ to stabilize. Four hundred $\mathrm{mL}$ of $95 \%$ ethanol (prepared from $100 \%$ anhydrous ethanol) was added to the 40 $\mathrm{L}$ aquarium to give a final concentration of $\sim 1 \%$ ethanol (vol/vol). After $\sim 60 \mathrm{~min}$, each fish was removed, and the electrode was allowed to return to ambient $\mathrm{PO}_{2}$ to allow for any correction of electrode drift. As a result, the exact time for each trial varied from 56-63 min and data are expressed as a percentage of that time to allow for ready comparison. At the end of each trial, fish were anesthetized in MS 222 and their intestines removed, sliced longitudinally, and the contents removed by gentle scraping. The removed contents were frozen at $-80^{\circ} \mathrm{C}$ until analysis.

\section{Microbial community profiling}

The intestinal contents of each fish were homogenized at room temperature in a microcentrifuge tube using a sterile pestle (Fisher Scientific, cat \# 12-141-364) and used for DNA extraction. The metagenomic DNA was extracted from intestinal contents of each fish using the QIAamp Fast DNA Stool Mini Kit and quantified using NanoDrop ND-1000. Microbial communities were profiled using V4 region of 16S rRNA gene and the sequencing was performed at Argonne National Laboratory. Briefly, V4 region of the 16S rRNA gene was PCR-amplified using modified primers 515F: GTGYCAGCMGCCGCGGTAA and 806R: GGACTACNVGGGTWTCTAAT, with the forward primer containing a 12 bp barcode [27]. The PCR products were cleaned, quantified and pooled at equimolar concentration. Paired-end sequencing of pooled amplicons was performed using MiSeq platform and customized sequencing primers.

\section{Data analysis}


A Welch's t-test administered in $\mathrm{R}$ was used to compare mean paradoxical anaerobism time in control and antibiotic-treated groups.

All sequence-based analysis was performed in QIIME2 (version 2018.6) [28] and the resulting data were exported for further analysis and visualization in R [29]. Raw Illumina reads were demultiplexed using sample-specific barcodes, denoised using DADA2 [30], and clustered into 938 high-quality ASVs. The DADA2 denoising approach was used to cluster sequences into ASVs as it can differentiate between true biological sequences from sequences containing errors introduced during amplification and sequencing, and simultaneously provides higher taxonomic resolution [30]. Taxonomy was assigned to each ASV using a naïve Bayesian classifier trained on the V4 region of 16S rRNA gene and the 'Silva 132 99\% OTUs full-length sequences' database. ANOSIM analysis was performed to identify differences in antibiotictreated and control group microbiomes. SIMPER analysis [31] was used to identify ASVs responsible for between-group differences. ANCOM analysis [32] was used to identify differentially abundant taxa.

Additionally, to assess the contribution of microbiome in ethanol metabolism, the potential microbial metagenome profile was obtained using PICRUSt [33]. For this, DADA2-denoised sequence data were used to pick OTUs (close-reference) using the VSEARCH algorithm and identified using the Greengenes database (version 13.5). The resulting OTU table was used as an input for PICRUSt analysis at http://galaxy.morganlangille.com/.

\section{List Of Abbreviations}

VDACs: Voltage-Dependent Anion Channels

rVO2: Rates of oxygen consumption

V4 region of $16 \mathrm{~S}$ rRNA: variable region 4 of $16 \mathrm{~S}$ ribosomal ribonucleic acid

bp: base pairs

PCR: Polymerase Chain Reaction

QIIME2: Quantitative Insights into Microbial Ecology 2

DADA2: Divisive Amplicon Denoising Algorithm 2

ASVs: Amplicon Sequence Variants

OTUs: Operational Taxonomic Units

NMDS: Nonlinear Multidimensional Scaling

ANOSIM: Analysis of Similarity

SIMPER: Similarity Percent 
ANCOM: Analysis of Composition of Microbiomes

VSEARCH: Vectorized search

PICRUSt: Phylogenetic Investigation of Communities by Reconstruction of Unobserved States

Adh: Alcohol dehydrogenase

adh: Alcohol dehydrogenase gene

NCBI: National Center for Biotechnology Information

SRA: Sequence Read Archive

$\mathrm{NAD}^{+}$: Nicotinamide Adenine Dinucleotide

\section{Declarations}

Ethics approval and consent to participate

All procedures were approved by the UNLV Institutional Animal Care and Use Committee.

\section{Consent for publication}

Not applicable.

\section{Availability of data and material}

The raw sequences are deposited in the NCBI Sequence Read Archive (SRA) under the BioProject accession PRJNA561361. All scripts used to process the 16S rRNA gene sequence data have been deposited in GitHub at https://github.com/hedlundb/PupFish

\section{Competing interests}

The authors declare that they have no competing interests.

\section{Funding}

This work was supported by a Faculty Opportunity Award from University of Nevada Las Vegas to FvB and SDH. FvB and BPH are supported by the National Science Foundation IOS 1655091 and IES 1516679 , respectively.

\section{Authors' contributions}

$\mathrm{FvB}, \mathrm{BPH}$, and SDH conceptualized the experiment. $\mathrm{BE}, \mathrm{MH}$, and $\mathrm{YM}$ carried out the oxygen rate measurements and collected the necessary samples for microbiota analysis. DF helped in DNA 
extraction. AF provided insights of presence of Cetobacterium in various desert springs in the Ash Meadow area. SSB carried out bioinformatics analysis and wrote the first draft of manuscript with the contribution from FvB and BPH. All authors reviewed the manuscript and approved the final version for submission.

\section{Acknowledgements}

Authors are thankful to the UNLV National Supercomputing Institute for allowing us to use Cherry Creek Cluster for performing the sequence analysis using QIIME2 pipeline. We are very thankful to Kevin Wilson, Corey Lee, and Jennifer Gumm for their support and guidance regarding the genetics of the fish used in these experiments and for general editorial advice.

\section{References}

1. Hillyard SD, Podrabsky J, van Breukelen Desert Environments. In: Riesch R, Tobler M, Plath M, editors. Extremophile fishes-ecology evolution and physiology of teleosts in extreme environments. New York: Springer; 2015. p. 59-84.

2. Heuton M, Ayala L, Burg C, Dayton K, McKenna K, Morante A, et al. Paradoxical anaerobism in desert pupfish. J Exp Biol. 2015;218:3739-45.

3. Heuton M, Ayala L, Morante A, Dayton K, Jones AC, Hunt JR, et al. Oxygen consumption of desert pupfish at ecologically relevant temperatures suggests a significant role for anaerobic metabolism. $J$ Comp Physiol B Biochem Syst Environ Physiol. 2018;188:821-30.

4. Lemasters JJ, Holmuhamedov E. Voltage-dependent anion channel (VDAC) as mitochondrial governator - Thinking outside the box. Biochim Biophys Acta - Mol Basis Dis. 2006;1762:181-90.

5. Lemasters JJ, Holmuhamedov EL, Czerny C, Zhong Z, Maldonado EN. Regulation of mitochondrial function by voltage dependent anion channels in ethanol metabolism and the Warburg effect. Biochim Biophys Acta - Biomembr. 2012;1818:1536-44.

6. Holmuhamedov EL, Czerny C, Beeson CC, Lemasters JJ. Ethanol suppresses ureagenesis in rat hepatocytes. J Biol Chem. 2012;287:7692-700.

7. Hochachka P, Somero G. Biochemical adaptation, mechanisms and process in physiological evolution. New York: Oxford University Press; 2002.

8. Shoubridge EA, Hochachka PW. Ethanol: Novel end product of vertebrate anaerobic metabolism. Science. 1980;209:308-9.

9. Holmuhamedov E, Lemasters JJ. Ethanol exposure decreases mitochondrial outer membrane permeability in cultured rat hepatocytes. Arch Biochem Biophys. 2009;481:226-33.

10. Tarnecki AM, Burgos FA, Ray CL, Arias CR. Fish intestinal microbiome: diversity and symbiosis unravelled by metagenomics. J Appl Microbiol. 2017;123:2-17.

11. Higuera-Llantén S, Vásquez-Ponce F, Barrientos-Espinoza B, Mardones FO, Marshall SH, OlivaresPacheco J. Extended antibiotic treatment in salmon farms select multiresistant gut bacteria with a 
high prevalence of antibiotic resistance genes. PLoS One. 2018;13(9): e0203641.

12. Sáenz JS, Marques TV, Barone RSC, Cyrino JEP, Kublik S, Nesme J, et al. Oral administration of antibiotics increased the potential mobility of bacterial resistance genes in the gut of the fish Piaractus mesopotamicus. Microbiome. 2019;7:24.

13. Loch TP, Faisal M. Emerging flavobacterial infections in fish: A review. J Adv Res. 2015;6:283-300.

14. Henríquez-Núñez $\mathrm{H}$, Evrard $\mathrm{O}$, Kronvall G, Avendaño-Herrera R. Antimicrobial susceptibility and plasmid profiles of Flavobacterium psychrophilum strains isolated in Chile. Aquaculture. 2012;354355:38-44.

15. Foster G, Ross HM, Naylor RD, Collins MD, Ramos CP, Garayzabal FF, et al. Cetobacterium ceti nov., sp. nov., a new Gram-negative obligate anaerobe from sea mammals. Lett Appl Microbiol. 1995;21:202-6.

16. Ray C, Bujan N, Tarnecki A, Davis DA, Browdy C, Arias CR. Analysis of the gut microbiome of Nile tilapia Oreochromis niloticus fed diets supplemented with Previda ${ }^{\circledR}$ and Saponin. J Fish. 2017; 11(2):36-45.

17. van Kessel MAHJ, Dutilh BE, Neveling K, Kwint MP, Veltman JA, Flik G, et al. Pyrosequencing of $16 \mathrm{~S}$ rRNA gene amplicons to study the microbiota in the gastrointestinal tract of carp (Cyprinus carpio). AMB Express. 2011;1:1-9.

18. Ramírez C, Coronado J, Silva A, Romero J. Cetobacterium is a major component of the microbiome of giant Amazonian fish (Arapaima gigas) in Ecuador. Animals. 2018;8(11):189.

19. Larsen AM, Mohammed HH, Arias CR. Characterization of the gut microbiota of three commercially valuable warmwater fish species. J Appl Microbiol. 2014;116:1396-404.

20. Sugita H, Miyajima C, Deguchi Y. The vitamin B12-producing ability of the intestinal microflora of freshwater fish. Aquaculture. 1991;92 C:267-76.

21. Schink B, Phelps TJ, Eichler B, Zeikus JG. Comparison of ethanol degradation pathways in anoxic freshwater environments. J Gen Microbiol. 1985;131:651-660.

22. Li F, Hinderberger J, Seedorf H, Zhang J, Buckel W, Thauer RK. Coupled ferredoxin and crotonyl coenzyme A (COA) reduction with NADH catalyzed by the butyryl-CoA dehydrogenase/Etf complex from Clostridium kluyveri. J Bacteriol. 2008;190:843-50.

23. Sackett JD, Huerta DC, Kruger BR, Hamilton-Brehm SD, Moser DP. A comparative study of prokaryotic diversity and physicochemical characteristics of devils hole and the ash meadows fish conservation facility, a constructed analog. PLoS One. 2018;13(3):e0194404.

24. Merrifield D, Ringo E. Aquaculture nutrition: gut health, probiotics and prebiotics. Wiley-Blackwell; 2014.

25. Burg GC, Johnson J, Spataro S, O'Keefe A, Urbina N, Puentedura G, et al. Care and propagation of captive pupfish from the genus Cyprinodon: insight into conservation. Environ Biol Fishes. 2019;102:1015-24. 
26. Mefferd CC, Bhute SS, Phan JR, Villarama JV, Do DM, Alarcia S, et al. A high-fat/high-protein, Atkinstype diet exacerbates Clostridioides (Clostridium) difficile infection in mice, whereas a highcarbohydrate diet protects. mSystems. 2020;5:e00765-19.

27. Caporaso JG, Lauber CL, Walters WA, Berg-Lyons D, Huntley J, Fierer N, et al. Ultra-high-throughput microbial community analysis on the Illumina HiSeq and MiSeq platforms. ISME J. 2012;6:1621-4.

28. Bolyen E, Rideout JR, Dillon MR, Bokulich NA, Abnet CC, Al-Ghalith GA, et al. Reproducible, interactive, scalable and extensible microbiome data science using QIIME 2. Nat Biotechnol. 2019;37:852-7.

29. The R Core Team. R: A Language and Environment for Statistical Computing. 2013;1.

30. Callahan BJ, McMurdie PJ, Rosen MJ, Han AW, Johnson AJA, Holmes SP. DADA2: High-resolution sample inference from Illumina amplicon data. Nat Methods. 2016;13:581-3.

31. Clarke KR. Non-parametric multivariate analyses of changes in community structure. Aust J Ecol. 1993;18:117-43.

32. Mandal S, Van Treuren W, White RA, Eggesb $\varnothing$ M, Knight R, Peddada SD. Analysis of composition of microbiomes: a novel method for studying microbial composition. Microb Ecol Heal Dis. 2015;26:27663.

33. Langille MGI, Zaneveld J, Caporaso JG, McDonald D, Knights D, Reyes J a, et al. Predictive functional profiling of microbial communities using 16S rRNA marker gene sequences. Nat Biotechnol. 2013;31:814-21.

34. Feuerbacher O, Bonar SA, Barrett PJ. Enhancing hatch rate and survival in laboratory-reared hybrid Devils Hole Pupfish through application of antibiotics to eggs and larvae. N Am J Aquacult. 2017; 79:106-114.

35. Martin AP. Genetic analysis of Cyprinodon diabolis. hybridization with nevadensis in the point of Rocks Refuge. Report submitted to the Nevada Division of Wildlife and the USFWS. 2005.

\section{Supplementary Figure Legend}

Fig. S1. Measurement of oxygen consumption. A schematic showing the arrangement of a glass metabolic chamber containing fish, an oxygen electrode, and peristaltic pump used to measure the oxygen consumption by pupfish.

\section{Figures}



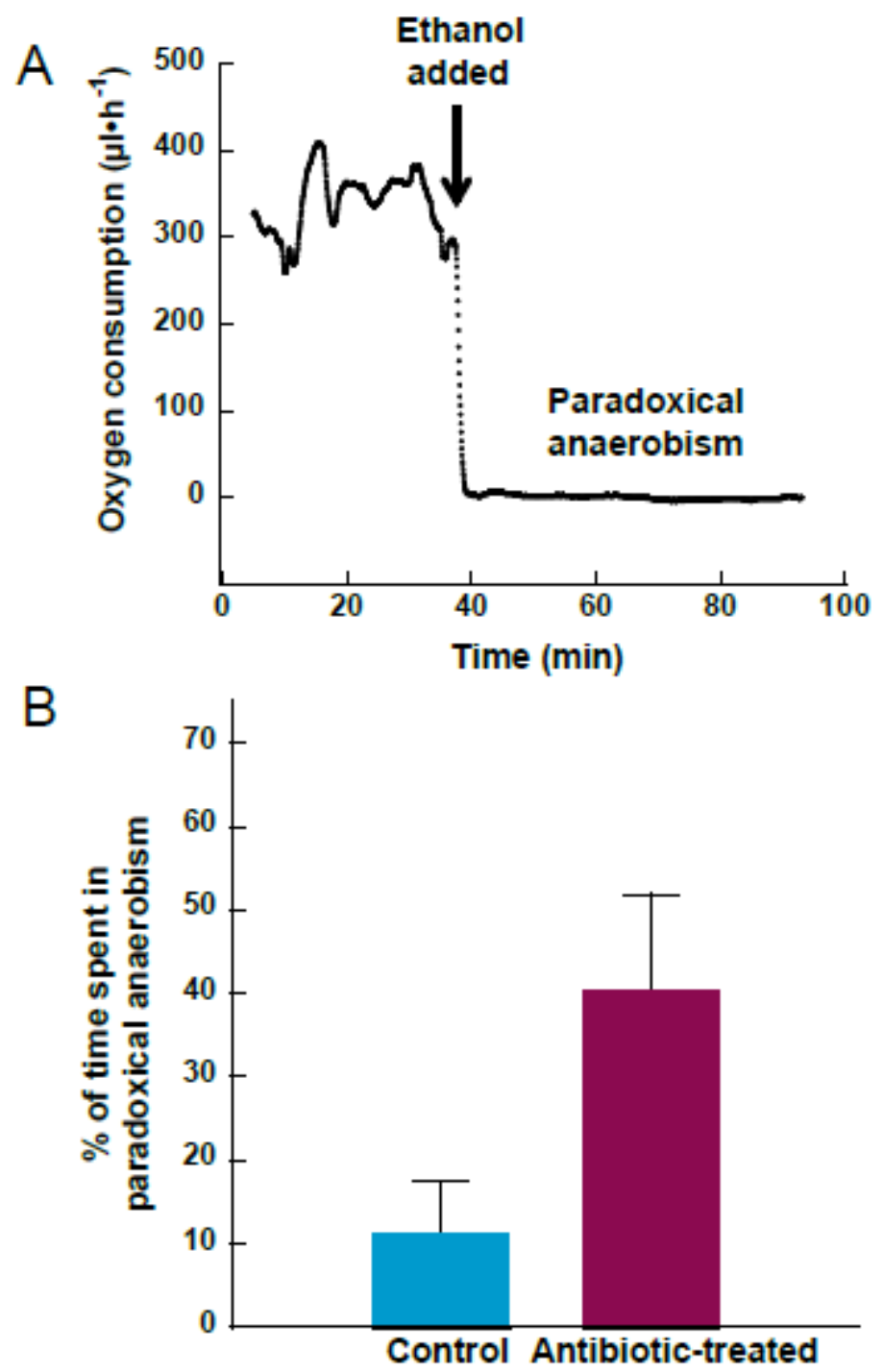

\section{Figure 1}

Measurements of paradoxical anaerobism. A) Demonstration of induction of paradoxical anaerobism using $1 \%$ ethanol (vol/vol). Pupfish ( $n=12$ antibiotic-treated pupfish and 12 control pupfish) were placed in the metabolic chamber and oxygen consumption was monitored. At the time indicated by the arrow, ethanol was added to a final concentration of $1 \%$ (vol/vol). In this example, an antibiotic-treated pupfish immediately entered paradoxical anaerobism and remained in that metabolic state for the duration of the experiment. B) Following addition of ethanol, pupfish oxygen consumption was monitored for $\sim 1 \mathrm{~h}$. Data are means \pm SE of the $\%$ of that time which was spent with negligible oxygen consumption. Values are significantly different (Welch's t-test, $p=0.037$ ). 
A

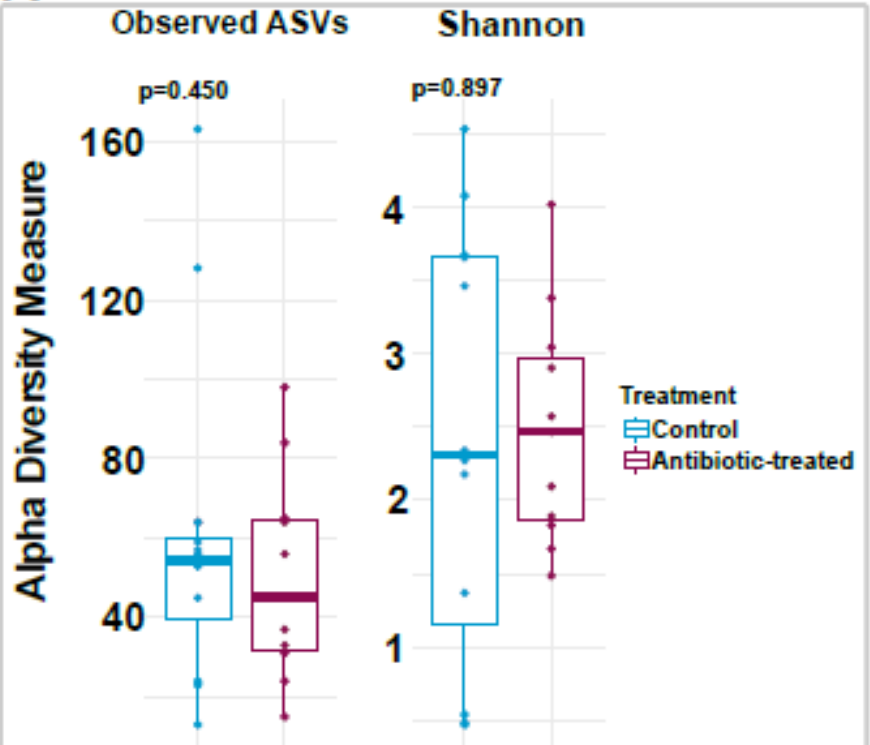

B

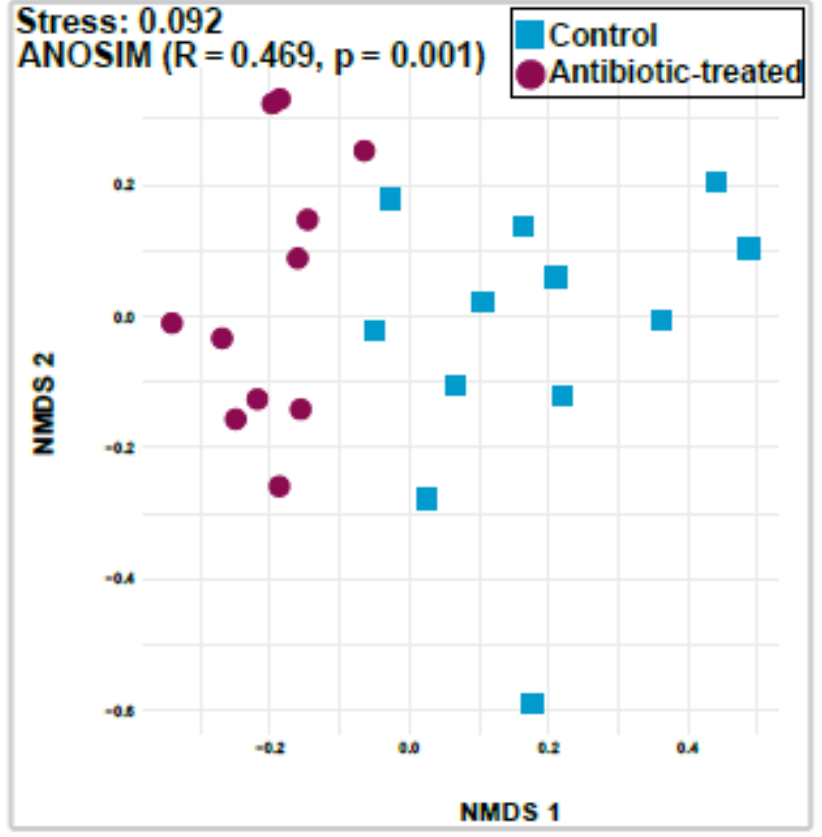

C

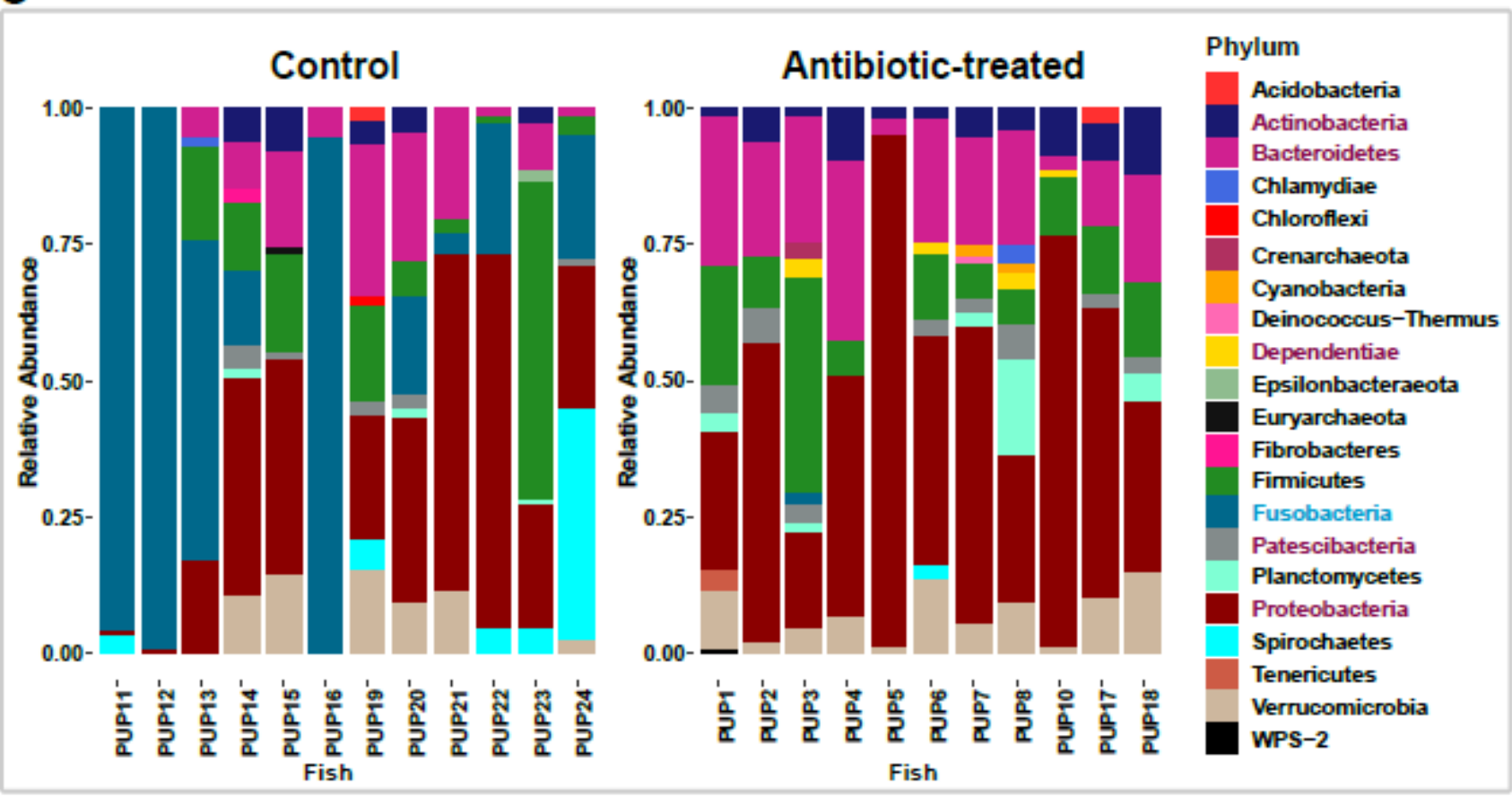

Figure 2

Microbial diversity analysis. A) Box-and-whisker plots of alpha diversity metrics (Observed ASVs and Shannon index). Each box represents interquartile range; the line inside the box is a median. B) BrayCurtis dissimilarity-based non-metric multidimensional scaling (NMDS) plot showing the distribution of antibiotic-treated and control fish samples in ordination space. C) Bar plot showing the relative abundance $(>1 \%)$ of gut microbiota at the phylum level. Five phyla indicated in cherry-red color in legend were significantly higher (Welch's t-test, $p<0.05$ ) in antibiotic-treated fish, whereas the one indicated in 
cyan (Fusobacteria) was significantly depleted (Welch's t-test, $\mathrm{p}<0.05$ ). The phylum Fusobacteria was dominated by genus Cetobacterium.

\section{Control}

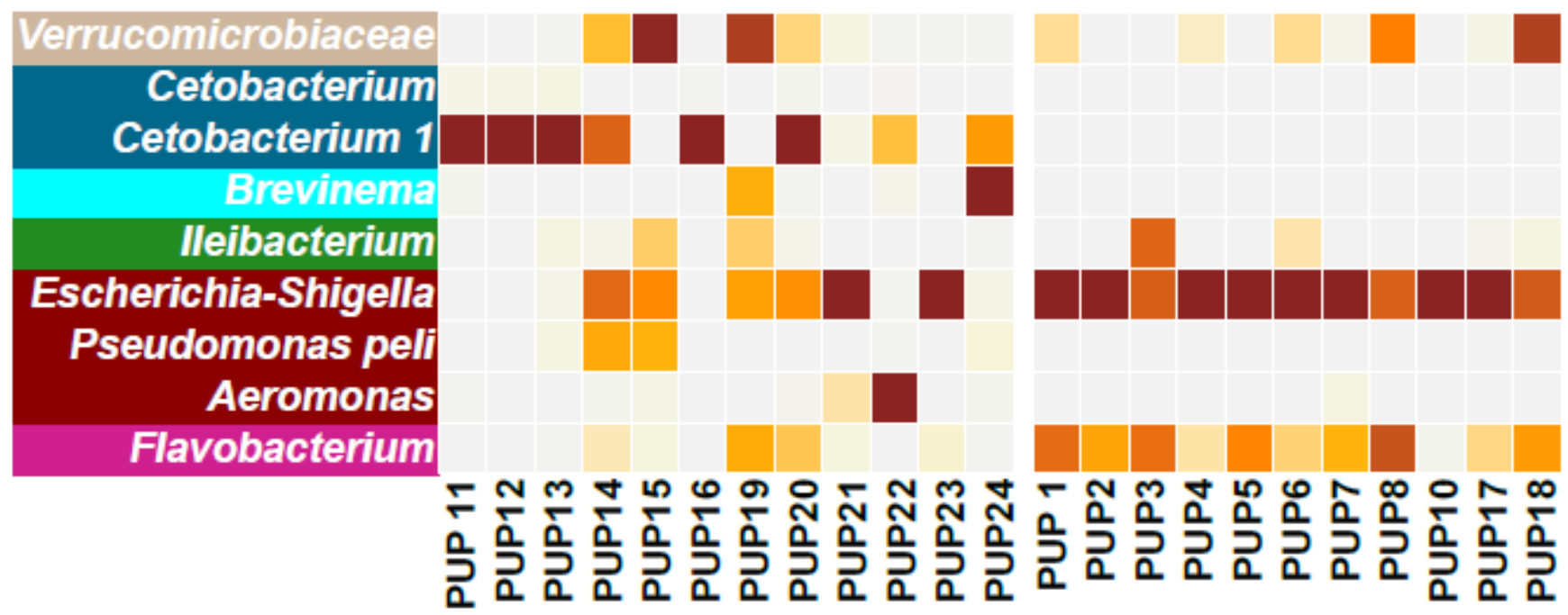

Figure 3

Heatmap displaying top ASVs responsible for dissimilarity between control and antibiotic-treated fish. Heatmap of the relative abundance of 09 ASVs (genus- or family-level assignments are indicated) identified using SIMPER analysis. These taxa contributed cumulatively to $50 \%$ of community dissimilarities. Each square represents the relative abundance of the given ASV, higher intensity of the brown color correlates with high relative abundance. Taxa are color-coded as per the phylum-level assignments as: Verrucomicrobia: orange; Fusobacteria: yellow; Spirochetes: pink; Firmicutes: sky-blue; Proteobacteria: green, and Bacteroidetes: grey.

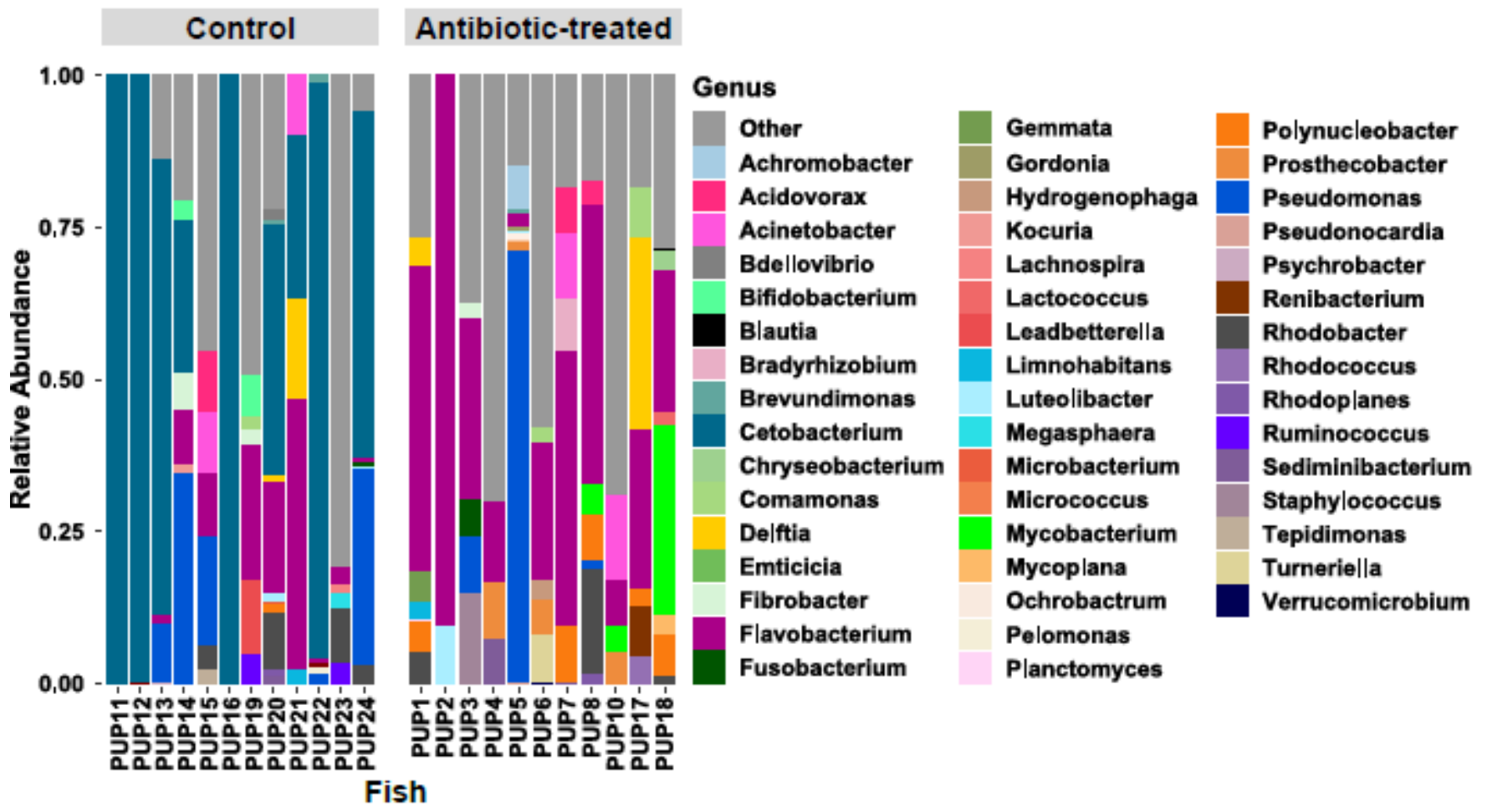


Figure 4

Genus-level relative abundance of 129 ASVs that are predicted to encode alcohol dehydrogenase. These ASVs are predicted to have alcohol dehydrogenase activity. ASVs with no predicted alcohol dehydrogenase are indicated as Other (red).

\section{Control}

\section{Antibiotic-treated}

Cetobacterium-rich community

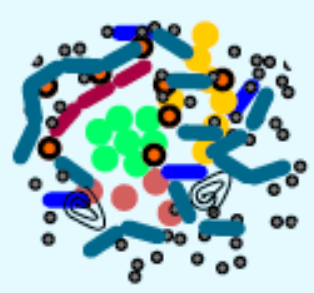

Cetobacterium is a possible ethanol sink

Low levels of ethanol reach fish tissues

Substrates can enter mitochondria

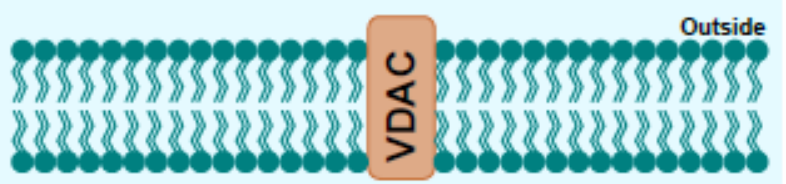

Fish continues with aerobic metabolism 
Summary of the results and hypothesis. We propose that the Cetobacterium in control pupfish may be acting as an ethanol sink. Thus, depleting Cetobacterium using the antibiotic cocktail might cause increased accumulation of the acetaldehyde in fish tissues leading to closure of VDAC and eventually the paradoxical anaerobism

\section{Supplementary Files}

This is a list of supplementary files associated with this preprint. Click to download.

- SupplementaryTables311.xlsx 